\title{
Controllable Synthesis, Structure and Property Modulation and Device Application of One-Dimensional \\ Nanomaterials
}


This page intentionally left blank 


\section{Controllable Synthesis, Structure and Property Modulation and Device Application of One-Dimensional Nanomaterials}

Proceedings of the 4th International Conference on One-Dimensional Nanomaterials (ICON2011)

Beijing, China,

7-9 December, 2011

Editor-in-chief

Yue Zhang

University of Science and Technology Beijing, China

Editors

Yousong Gu

Yunhua Huang

Xiaoqin Yan

Qingliang Liao

University of Science and Technology Beijing, China 


\section{Published by}

World Scientific Publishing Co. Pte. Ltd.

5 Toh Tuck Link, Singapore 596224

USA office: 27 Warren Street, Suite 401-402, Hackensack, NJ 07601

UK office: 57 Shelton Street, Covent Garden, London WC2H 9HE

\section{British Library Cataloguing-in-Publication Data}

A catalogue record for this book is available from the British Library.

\section{CONTROLLABLE SYNTHESIS, STRUCTURE AND PROPERTY MODULATION AND DEVICE APPLICATION OF ONE-DIMENSIONAL NANOMATERIALS Proceedings of the 4th International Conference on One-Dimensional Nanomaterials (ICON2011)}

Copyright () 2012 by World Scientific Publishing Co. Pte. Ltd.

All rights reserved. This book, or parts thereof, may not be reproduced in any form or by any means, electronic or mechanical, including photocopying, recording or any information storage and retrieval system now known or to be invented, without written permission from the Publisher.

For photocopying of material in this volume, please pay a copying fee through the Copyright Clearance Center, Inc., 222 Rosewood Drive, Danvers, MA 01923, USA. In this case permission to photocopy is not required from the publisher.

ISBN-13 978-981-4407-59-5

ISBN-10 981-4407-59-3

Printed in Singapore. 


\section{Preface}

The 4th International Conference on One-dimensional Nanomaterials (ICON2011) was held successfully from December 7 to 9, 2011 in Beijing, China. This conference provides a platform for communications among researchers in the field of one-dimensional nanomaterials to exchange ideas, present achievements and discuss the future of one-dimensional nanomaterials. It is a great get together for top experts in the world in the field of one dimensional nanomaterial. It can serve the interest of our scientific communities, as well as industries and general public at large.

ICONs were held successfully in Chinese Taipei in 2005, Sweden in 2007 and USA in 2009. It's the first time for ICON to come to mainland China and we are delight to see a great increase in participants. There are 8 keynote speakers, 24 invited speakers and 36 oral presenters and 67 posters.

In recent years, rapid progresses have been made in both fundamental research and technique applications of one-dimensional nanomaterials such as nanowires, nanofibers, nanobelts, nanorods, etc. due to their remarkable physical and chemical properties as well as wide potential in applications. Rational design and controllable production of one-dimensional nanostructures are the basis for optimization the performance of nanoscale devices and systems for application in electronics, optoelectronics and spintronics etc.

The conference focused on the rational synthesis, structure modulation and property optimization, device fabrication, system integration and novel applications of 1D nanomaterials in nanoelectronics, nano-optoelectronics, nanophotonics, nanopiezotronics, biomedical sciences, sensors, energy technology (solar cell, thermoelectric, piezoelectric nanogenerator, energy storage), and environmental sciences.

The proceedings of the conference are divided into 8 sections: (1) Growth mechmics and structure modulation, (2) property modulations, (3) theoretical simuluaitons, (4) photoic devices, (5) bio-sensors, (6) mechanical detectors (7) solar cells (8) nanodamage. 
We would like to give our sincere thanks to all of our new and old friends, who have come to Beijing to attend the conference, and special thanks to the authors who contribute their papers to the conference proceedings.

We would like to thank Ph.D students Zi Qin, Xiaohui Zhang, Zheng Zhang, Qi Zhang, Yanguang Zhao, Guangjie Zhang. They spent a lot of time on proof reading the contributed papers, checking and correcting the content, language and format.

This conference was sponsored by the National Natural Science Foundation of China, the Ministry of Education of the People's Republic of China, University of Science and Technology Beijing, FEI Company, and JEOL Ltd, etc.

Yue Zhang University of Science and Technology Beijing 


\title{
The 4th International Conference on One-dimensional Nanomaterials
}

\author{
Bejing 7-9 December, 2011
}

\section{Organizer}

Research Center for Nanoscience and Technology,

University of Science and Technology Beijing, China

\section{Sponsors}

National Natural Science Foundation of China

Ministry of Education of the People's Republic of China

University of Science and Technology Beijing

FEI Company

Advisory Board (Alphabetical)

Prof. Yoshio Bando

National Institute for Materials Science, Japan

Prof. Shoushan Fan

Tsinghua University, China

Prof. Ruiping Gao

National Natural Science Foundation of China, China

Prof. Runsheng Gao

Ministry of Education of China, China

Prof. Minghong He

National Natural Science Foundation of China, China

Prof. Ming Li

National Natural Science Foundation of China, China

Prof. Charles M. Lieber

Harvard University, USA

Prof. Zhongfan Liu

Peking University, China

Prof. $\mathrm{Ke} \mathrm{Lu}$

Institute of Metal Research, CAS, China

Prof. Chong-Yun Park

Sungkyunkwan University, Korea

Prof. Lars Samuelson

Lund University, Sweden

Prof. Sishen Xie

Institute of Physics, CAS, China

Prof. Ningsheng Xu

Sun Yat-sen University, China

Prof. Qikun Xue

Tsinghua University, China

Prof. Jing Zhu

Tsinghua University, China

Prof. Xing Zhu

Peking University, China 
viii

\section{Conference Chairs}

Prof. Ze Zhang

Prof. Yue Zhang

Prof. Zhonglin Wang
Zhejiang University, China

University of Science \& Technology Beijing, China

Georgia Institute of Technology, USA

\section{Organizing Committee (Alphabetical)}

Prof. Xuedong Bai

Prof. Kexin Chen

Prof. Huiming Cheng

Prof. Le Si Dang

Prof. Hongjin Fan

Prof. Lin Guo

Prof. Wanlin Guo

Prof. Xiaodong Han

Prof. Zheng $\mathrm{Hu}$

Prof. Sang-Woo Kim

Prof. Cheol-Jin Lee

Prof. Liwei Lin

Prof. Yichun Liu

Prof. Sanjay Mathur

Prof. Guowen Meng

Prof. Lianmao Peng

Prof. Chen Wang

Prof. Dapeng Yu

Prof. Shuhong Yu

Prof. Magnus Willander
Institute of Physics, CAS, China

National Natural Science Foundation of China, China

Institute of Metal Research, CAS, China

CNRS and Université de Grenoble, France

Nanyang Technological University, Singapore

Beihang University, China

Nanjing University of Aeronautics \& Astronautics, China

Beijing University of Technology, China

Nanjing University, China

Sungkyunkwan University, Korea

Korea University, Korea

University of California at Berkeley, USA

Northeast Normal University, China

University of Cologne, Germany

Institute of Solid State Physics, CAS, China

Peking University, China

National Center for Nanoscience\& Technology, China

Peking University, China

University of Science \& Technology of China

Linköping University, Sweden 


\section{Contents}

Preface $\quad$ v

Conference Committee vii

Graphene and Graphene-Based Nanocomposites: Synthesis and $\quad 1$ Supercapacitor Applications

G. K. Wang, C. S. Liu, X. Sun, F. Y. Lu, H. T. Sun and J. Lian

Progress in the Hydrothermal Formation of Dispersive Nano-Particles and Whiskers

L. Xiang

The Growth of Al-Doped ZnO Nanoplate Arrays Influenced by Solution Concentration

L. D. Tang and B. Wang

Influences of Hydrothermal Conditions on Morphologies of $\mathrm{ZnO}$

Nanowire Arrays

Z. Qin, Y. H. Huang, Q. Y. Wang and G. J. Zhang

Microstructure and Growth Mechanism of Mn Doped ZnS Nanobelts

J. J. Qi, Q. Zhang, Z. Q. Deng and Y. Zhang

Shape-Dependent Surface Plasmon Resonance of Ag Nanocrystallines in OPAA Template

S. S. Han and X. C. Yang

Electrical Properties and Electric-Induced Nanodamage of Single

Crystalline Bead-Shaped ZnO Nanorod

H. F. Li, P. F. Li and Y. H. Huang

Optical Properties and Photocatalytic Activity of Mn-Doped ZnO Nanorods

J. Zhao, Z. M. Bai, X. Q. Yan and L. Wang 
Structure and Magnetic Property of Ni-Doped ZnO Nanorods

X. H. Zhang, X. Q. Yan, Q. L. Liao and J. Zhao

Doping Effect of the Electronic Transport Properties of Zinc Oxides

Nanowires Studied by First Principles Calculation

Y. S. Gu, X. Sun, X. Q. Wang and Y. Zhang

Elastic Response of Copper Slabs to Biaxial Strain Studied by Density

Functional Theory Calculation

X. Q. Wang, Y. S. Gu and X. Su

Electronic Transport Properties of One Dimensional ZnO Nanowires

Studied Using Maximally-Localized Wannier Functions

X. Sun, Y. S. Gu and X. Q. Wang

Site Dependent Transport Properties of N-Doped Graphene Nanoribbons

with Zigzag Edges

Y. Hu, Y. S. Gu, X. Sun and X. Q. Wang

Length Effect on the Electronic Transport Properties of $\mathrm{Mg} / \mathrm{ZnO}$

NW/Mg Nanostructures Studied by First Principles Calculation

Y. S. Gu, R. Gao, X. Sun and X. Q. Wang

Ordered $\mathrm{ZnO}$ Nanorod Arrays for Ultraviolet Detection

F. Yi, Y. H. Huang and Y. S. Gu

Effect of Localized UV Irradiation on Transport Property in $\mathrm{ZnO}$

Nanotetrapod Devices

W. H. Wang, J. J. Qi, Q. Y. Wang, Z. Qin, Z. Z. Wang, X. Sun and $F . Y i$

ZnO Nanowires Based MSM Ultraviolet Photodetectors with Pt Contact Electrodes

H. S. Liu, X. Q. Yan, S. W. Ma and Z. M. Bai

Solution Processed ZnO Nanorod Arrays/PFO Hybrid Heterojunction for Light Emitting

H. S. Liu, X. Q. Yan, X. Chen and Y. S. Gu 
Fabrication and Performance Study on Individual $\mathrm{ZnO}$ Nanowires Based Bioelectrode

Y. G. Zhao and X. Q. Yan

Enzyme-Based Lactic Acid Detection Using AlGaAs/GaAs High

Electron Mobility Transistor with Sb-Doped ZnO Nanowires Grown on the Gate Region

S. W. Ma, Y. H. Huang, H. S. Liu, X. H. Zhang and Q. L. Liao

Single ZnO Nanowire-Based BioFET Sensors for Ultrasensitive, LabelFree and Real-Time Detection of Uric Acid

P. Lin, X. Liu, X. Q. Yan, Z. Kang, Y. Lei and Y. G. Zhao

A High-Performance Glucose Biosensor Based on ZnO Nanorod Arrays 162 Modified with Au Nanoparticles

G. Zhang, Y. Lei and X. Q. Yan

Piezotronic Vibration Detector Based on $\mathrm{ZnO}$ Nanowire Arrays

Z. Zhang, Y. H. Huang, Q. L. Liao, P. Li and S. P. Chen

Fabrication and Properties of a Microstrain Sensor Based on Zinc Oxide 181 Network Structure

P. Li, Q. L. Liao, Z. Zhang, S. W. Ma and Y. Zhang

Strain Sensors Based on Single High-Quality ZnO Microwires

Z. W. Liu, X. Q. Yan and Y. Zhang

A Design of Rainbow Solar Cell: An Orderly Gradient of CdS-CdSe

Sensitized ZnO Solar Cell

X. Y. Hu and Y. W. Tang

Fabrication of Good Quality N (a-Si) - (na-Si)-P (mc-Si) Tunnel

Junction for Tandem Solar Cells

M. J. Shi, Y. Zhang and L. L. Chen

Preparation of Ga-Doped ZnO Nanorod Arrays for Dye Sensitized Solar Cells Applications

Y. Qiu, Z. Qin and Y. S. Gu 
xii

Controlled Synthesis of Zno Nanotetrapods and Performance OF Zno Nanotetrapods Based Dye-Sensitized Solar Cells

Q. Pang, Y. J. Feng, C. J. Liang And J. He

Electromagnetic and Microwave Absorption Properties of Carbonyl Iron/Tetrapod-Shaped $\mathrm{ZnO}$ Nanostructures Composite Coatings

H. B. Yu, H. Qin and Y. H. Huang

Effect of Corrosion by Diluted $\mathrm{HCl}$ Solution on the $\mathrm{ZnO}$ : Al Texture

M. J. Shi, P. Wang and L. L. Chen

Nanodamage and Nanofailure of $1 \mathrm{D} \mathrm{ZnO}$ Nanomaterials and

Nanodevices

P. F. Li, Y. Yang, Y. H. Huang and Y. Zhang

Author Index 